\title{
Efeitos do tratamento com metformina no crescimento de células do carcinossarcoma de Walker 256
}

\author{
Bianca F. Ehmke (IC), André Gustavo de Oliveira (PG), Maria Cristina C. Gomes-Marcondes (PQ).
}

\section{Resumo}

A caquexia causa grande perda de peso dos pacientes com câncer, reduzindo a qualidade e a expectativa de vida. O uso de metformina, em animais com câncer, mostra resultados interessantes, como diminuição da proliferação celular e apoptose. Considerando o câncer como a segunda maior causa de morte inclusive no Brasil e o alto grau de mortalidade associadas ao processo de caquexia, esse estudo analisou os benefícios da administração de metformina em animais portadores do tumor de Walker-256, diminuindo a proliferação celular ou aumentando apoptose e, assim, diminuindo o estado caquético dos animais.

Palavras Chave: Câncer, Caquexia, Metformina

\section{Introdução}

O estado de câncer-caquexia caracteriza-se
pela intensa mobilização de nutrientes e
disponibilidade desses à atividade celular
neoplásica (1). O tumor de Walker 256 é um
modelo experimental de caquexia (2) produzindo
alterações bioquímicas e metabólicas no
hospedeiro, semelhantes às encontradas em
pacientes. Dessa forma, a manutenção da massa
magra é um dos principais fatores para melhora
no prognóstico de pacientes com câncer-
caquexia, sendo que quanto maior for a perda de
peso, menor é o tempo de sobrevida (3).
Metformina (N,N-dimetilbiguanida) é um fármaco
administrado para pacientes com diabetes
mellitus tipo 2 (DMT2) (4) e também mostra efeito
antiproliferativo sobre o desenvolvimento tumoral (5).

Esse trabalho avaliou os efeitos moduladores da metformina sobre o crescimento do tumor Walker256 e seus efeitos em ratos. Analisou-se 5 grupos de ratos (cada grupo com 5 ou 6 animais) distribuídos: Controle (C), Metformina (M), Tumor de Walker (W), Tumor de Walker com tratamento de metformina desde a inoculação tumoral (WM) e Tumor de Walker com tratamento de metformina após o tumor ficar palpável (WpM), durante 30 dias de evolução tumoral.

\section{Resultados e Discussão}

Analisou-se 0 peso relativo dos órgãos, observando aumento no fígado, baço e adrenal, como também elevada porcentagem de agua corpórea de todos os grupos W, WM e WpM, quando comparados aos controles. Houve diminuição da gordura inguinal e perirenal nos animais com tumor. Houve intensa espoliação da massa magra nos animais com tumor em relação aos grupos controle (C e M), em função da mobilização de nutrientes para prover a atividade celular neoplásica (1). Associada ao crescimento tumoral, a caquexia relaciona-se a perda involuntária de peso (2) principalmente da massa magra e gordura.

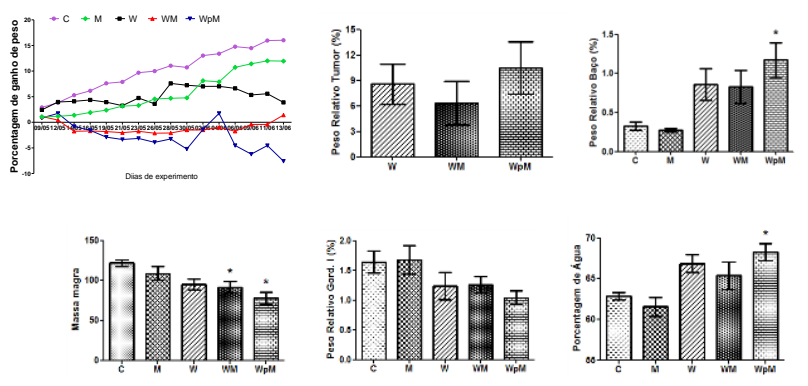

Conclusões

Conclui-se que os animais com tumor (W, WM e WpM) apresentaram o desenvolvimento do estado caquético com menor tempo de sobrevida do que os animais dos grupos controle (C e M).

\section{Agradecimentos}

Fapesp 2013/16115-1; 2010/00209-9. CNPq 302863/2013-3; CNPq-PIBIC/UNICAMP.

1. Tisdale MJ. Metabolic abnormalities in cachexia and anorexia. Nutrition. 2000;16(10):1013-4.

2. Emery PW. Cachexia in experimental models. Nutrition. 1999;15(7-8):600-3.

3. Tisdale MJ. Mechanisms of cancer cachexia. Physiological reviews. 2009;89(2):381-410.

4. Turner RC, Holman RR. Metformin and risk of cardiovascular disease. Cardiology. 1999;91(3):203-

5. Oliveira AG, Gomes-Marcondes MCC (2014) Metformin Improves Carbohydrate Metabolism and Minimizes Walker Tumor Growth in Young Rats. Biochem Pharmacol 3:125. doi: $10.4172 / 2167-0501.1000125$ 